\title{
Service Publishing and Discovering Model in a Web Services Oriented Peer-to-Peer System*
}

\author{
Ruixuan Li, Zhi Zhang, Wei Song, Feng Ke, and Zhengding Lu \\ College of Computer Science and Technology, \\ Huazhong University of Science and Technology, Wuhan 430074, Hubei, P.R. China \\ rxli@public.wh.hb.cn, wustzz@sina.com, sw_cyt@126.com, \\ michael_ke@163.com, zdlu@mail.hust.edu.cn
}

\begin{abstract}
To enhance the reliability and scalability of the service oriented architecture, this paper introduces a Web Services Oriented Peer-to-peer (WSOP) architecture with a combination of centralized and decentralized characteristics, and gives a framework of service publishing and discovery model based on WSOP architecture.
\end{abstract}

\section{Introduction}

Web Services and peer-to-peer technologies widely emerged during the last several years and these two diagrams tend to be polled together in the recent researches. Recent work in content-based search include content-addressable networks - where the content of queries is used to efficiently route messages to the most relevant peers as well as some variations of publish/subscribe networks [1]. These content-based P2P networks place emphasis on locating and distributing the contents rather than on logical organization of the system architecture and on publishing and discovering Web Services through the peer-to-peer network. Several other projects concern the combination of web services and peer-to-peer networks where [2] gives a general overview and a classification of P2P based web services. This paper introduces Web Services Oriented Peer-to-peer (WSOP) architecture with a combination of centralized and decentralized characteristics, and presents a framework of Web Services publishing and discovery model based on WSOP architecture.

\section{Web Services Oriented Peer-to-peer (WSOP) Architecture}

We present a Web Services Oriented Peer-to-peer (WSOP) architecture based on the integration of different peer-to-peer systems and Common Web Services (CWS) with the SOAP (Simple Object Access Protocol) connectivity (see Fig. 1). In our approach, peers residing as the neighbors (e.g. with the same interests) on the same P2P network are pulled together to form a peer group. There is at least one super peer in each peer group. It maintains a Local Service Registry Broker (LSRB), providing the fast service registration and invocation in the peer group environment. The CWS, hosted on the SOAP server, consists of service provider, service requestor and Com-

* This work was partially supported by National Natural Science Foundation of China under Grant 60403027, National Key Technologies R\&D Program of China under Grant 2002BA103A04 
mon Service Registry Broker (CSRB). The CSRB provides access to a P2P network interconnecting nodes (i.e. super peers) in different physical networks using different transport protocols and maintains the mappings of service descriptions between CSRB and LSRB. An important advantage of this architecture would be the flexibility of registering new services to the system via employing the super peers and LSRBs. It will definitely reduce the heavy load of the CSRB when the number of nodes and services in the environment are very large. Another advantage is that service request delivering would not be flooded in the whole systems.

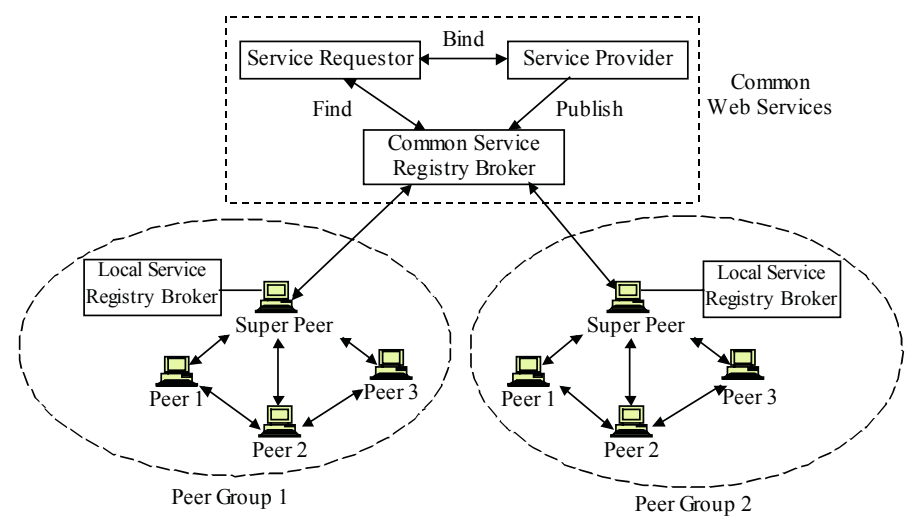

Fig. 1. Web Services oriented peer-to-peer (WSOP) architecture

\section{Service Publishing and Discovery Model Based on WSOP Architecture}

In traditional manner, the mechanism of service publishing and discovery are centralized through using common UDDI. The WSOP-based architecture will employ the peer-to-peer technologies and decentralize the UDDI service directory. Each peer in the P2P overlay network plays the roles of service provider and service consumer. The super peers in the peer group will take the responsibility for service registry in the group. So, the peer will take most of the work for service publishing and discovery. Fig. 2 illustrates the framework of service publishing and discovery model based on WSOP architecture.

The framework includes several modules, such as P2P System Initialization, Peer Group Discovery, Peer Authentication, Web Services Configuration, WSDL Processing. Service Publishing module uses a service advertisement to publish a service the Export Pipes and the peers can cache the published Web Services advertisements. The Service Discovery module will look up the discovered Web Services advertisements in the local service cache and discover new Web Services advertisements in the WSOP-based system through using static and dynamic discovery methods. It also can cache the discovered valuable Web Services advertisements. Once the services are discovered, the peer can invoke and utilize the service through WSIF (Web Services Invocation Framework) protocol. It uses the same programming model whatever the Web Services are implemented through using WSIF protocol. It can also access the services dynamically generated without stubs. 


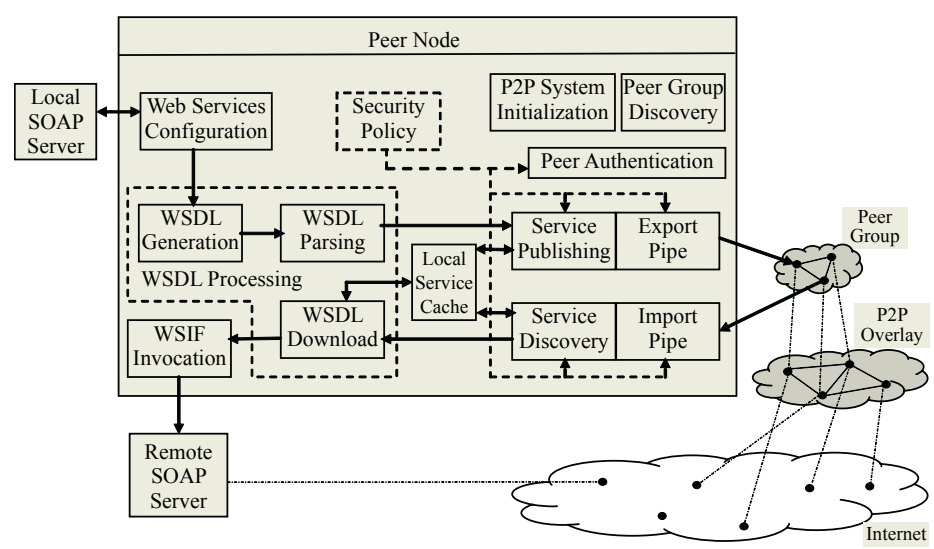

Fig. 2. The framework of service publishing and discovery model based on WSOP architecture

\section{Conclusions}

In this paper we highlighted key intersect points that enable using Web Services infrastructure and peer-to-peer technologies together and presented an architectural approach and infrastructure towards unifying them. There are several advantages that the service publishing and discovery model based on WSOP architecture offers. These include simplicity and ease of use, openness, reliability, scalability and security.

\section{References}

1. Papazoglou, M. P., Kramer, B. J., Yang, J.: Leveraging Web-Services and Peer-to-Peer Networks. In: Proceedings of the 15th International Conference on Advanced Information Systems Engineering(CaiSE2003), Lecture Notes in Computer Science, Vol. 2681. Springer-Verlag, Berlin Heidelberg New York (2003) 485-501

2. Schmidt, C., Parashar, M.: A Peer-to-Peer Approach to Web Service Discovery. World Wide Web archive, Vol. 7(2) (2004) 211-229 